\title{
Mindfulness practice for protecting mental health during the COVID-19 pandemic
}

\author{
Julie Lei Zhu', Rasmus Schülke $\mathbb{B}^{2}$, Deniz Vatansever ${ }^{2}$, Dayou Xi ${ }^{3}$, Junjie Yan ${ }^{4}$, Hanqing Zhao ${ }^{4}$, Xiaohua Xie ${ }^{2}$, \\ Jianfeng Feng ${ }^{2}$, Mark Yuting Chen ${ }^{4}$, Barbara Jacquelyn Sahakian $\mathbb{0}^{5}$ and Shouyan Wang $\mathbb{0}^{2}$
}

\begin{abstract}
Emerging evidence shows that the coronavirus disease 2019 (COVID-19) pandemic is negatively affecting mental health around the globe. Interventions to alleviate the psychological impact of the pandemic are urgently needed. Whether mindfulness practice may protect against the harmful emotional effects of a pandemic crisis remains hitherto unknown. We investigated the influence of mindfulness training on mental health during the COVID-19 outbreak in China. We hypothesized that mindfulness practitioners might manifest less pandemic-related distress, depression, anxiety, and stress than non-practitioners and that more frequent practice would be associated with an improvement in mental health during the pandemic. Therefore, we assessed pandemic-related distress and symptoms of depression, anxiety, and stress, as well as the frequency of meditation practice at the peak of new infections (Feb 4-5; $N=673$ ) and three weeks later (Feb 29-30; $N=521$ ) in mindfulness practitioners via online questionnaires. Self-reported symptoms were also collected from non-practitioners at peak time only $(N=1550)$. We found lower scores of pandemic-related distress in mindfulness practitioners compared to non-practitioners. In general, older participants showed fewer symptoms of depression and anxiety. In younger practitioners, pandemic-related distress decreased from peak to follow-up. Importantly, increased mindfulness training during the preceding two weeks was associated with lower scores of depression and anxiety at both assessments. Likewise, practice frequency predicted individual improvement in scores of depression, anxiety, and stress at follow-up. Our results indicate that mindfulness meditation might be a viable low-cost intervention to mitigate the psychological impact of the COVID-19 crisis and future pandemics.
\end{abstract}

\section{Introduction}

Mental disorders are a leading cause of disability with extensive socio-economic consequences. Conditions such as major depressive and anxiety disorders have a considerable impact on large portions of the population, with estimated global prevalences of $4.4 \%$ and $3.6 \%$, respectively, in $2015^{1}$. Although a complex interaction of both biological and environmental influences gives rise to mental illness, a common risk factor that has long been

\footnotetext{
Correspondence: Barbara Jacquelyn Sahakian (bjs1001@cam.ac.uk) or Shouyan Wang (shouyan@fudan.edu.cn)

${ }^{1}$ Fanhai International School of Finance, Fudan University, Shanghai, China ${ }^{2}$ Institute of Science and Technology for Brain-Inspired Intelligence, Fudan University, Shanghai, China

Full list of author information is available at the end of the article

These authors contributed equally: Julie Lei Zhu, Rasmus Schülke
}

identified is stress ${ }^{2}$. Stress triggered by major life events plays a pivotal role in the emergence of depressive symptoms, which are often proportional to the scale of the events and whether they involve interpersonal loss or social rejection ${ }^{3}$. Moreover, social isolation and loneliness are linked to higher levels of depressive symptoms ${ }^{4-6}$. Finally, global events like natural disasters, technological disasters, and terrorist acts severely increase the risk of depression across large populations ${ }^{7}$, necessitating specific and wide-scale interventions to alleviate their impact on mental health ${ }^{8,9}$.

A converging body of evidence suggests that the current viral outbreak may also act as a severe external stressor and have deleterious effects on public mental health ${ }^{10}$. The COVID-19 pandemic has given rise to fear 
concerning the disease's health risks (for oneself and one's loved ones) $)^{11,12}$ and its long-term economic consequences ${ }^{12,13}$. Furthermore, a recent review found that quarantine measures may increase symptoms of acute and post-traumatic stress, depression, and anger ${ }^{14}$.

A number of studies around the world have focused on assessing measures of stress, distress, depression, and anxiety across large population samples during the current COVID-19 outbreak. 54\% of respondents in a survey conducted at the peak of the pandemic in China rated the psychological impact of the pandemic as moderate to severe, with $29 \%$ reporting moderate to severe symptoms of anxiety ${ }^{15}$. Two studies looked specifically at COVID-19 related distress and found mild to moderate distress in $29 \%$ and severe distress in $5.1 \%$ of Chinese respondents ${ }^{16}$, as well as $47 \%$ of mild to moderate distress and $14.1 \%$ of severe distress in Iranian respondents ${ }^{17}$. Likewise, an Italian survey conducted three weeks into the COVID-19 lockdown measures found high rates of symptoms of post-traumatic stress disorder, depression, anxiety, insomnia, and stress, especially in the case of COVIDrelated stressful life events, and discontinued working activity $^{18}$. In another Italian study, students reported more depressive symptoms during lockdown compared to six months before ${ }^{19}$.

These studies highlight that mental health during pandemics is not only impacted in vulnerable groups like medical staff ${ }^{20-22}$ and patients with mental disorders ${ }^{23}$, but also in the general population. Therefore, protective factors and interventions to improve public mental health during global pandemics need to be identified and developed $^{24,25}$. So far, factors such as continuing to work actively during the pandemic either in the usual workplace or home office ${ }^{18,26}$, physical exercise ${ }^{26}$ and limited media exposure ${ }^{12,26}$ have been linked to lower degrees of anxiety, depression, and stress and could thus be useful for mitigating the psychological impact of the current and future pandemic crises. However, the potential influence of daily practices directly aimed at improving mental health, such as mindfulness meditation, hitherto remains unknown.

Recently, mindfulness training interventions have emerged as a promising approach to foster mental health ${ }^{27}$. Originally based on ancient contemplative traditions, modern mindfulness interventions combine practices of relaxation and meditation into structured training, sometimes incorporating further elements of cognitive-behavioral therapy ${ }^{28}$. While specific implementations of mindfulness practices differ, these techniques usually involve the repeated practice of non-judgmental observation, in order to achieve mental stability and a state of mindfulness that is characterized by relaxed vigilance for distractions ${ }^{27}$. It has been shown in meta-analyses that mindfulness-based therapies are effective at reducing symptoms of depression and anxiety ${ }^{29,30}$. Mindfulness-based cognitive therapy has been validated as a clinical treatment for relapse prevention in recurrent depression $^{31}$, was shown to be effective for treating current depressive symptoms ${ }^{32}$, and is being recommended for preventing depressive relapse by the United Kingdom's National Institute for Health and Care Excellence ${ }^{33}$. In addition, mindfulness-based interventions reduce psychological stress ${ }^{34}$ by fostering stress resilience ${ }^{35}$ and are effective at reducing social anxiety disorder ${ }^{36}$. Mindfulness-based interventions are not only effective in clinical populations, but also reduce symptoms of distress, depression, anxiety, and stress in otherwise healthy individuals ${ }^{37}$. Mindfulness training may be especially beneficial in populations exposed to high levels of stress ${ }^{38-40}$. Previously, research centered on designing mindfulness interventions ${ }^{41,42}$ and elucidating its neural mechanisms ${ }^{43,44}$. Most studies on the efficacy of mindfulness treatments to alleviate symptoms of mental disorders have been conducted in research settings, and few investigations have focused on their impact in actual clinical practice or real-life environments ${ }^{27}$.

In this observational study, we investigated whether mindfulness practice under lockdown conditions during the COVID-19 pandemic might be associated with a change in mental health. First, we compared measures of mental health between mindfulness practitioners and nonpractitioners at the peak of the COVID-19 pandemic in China, controlling for individual differences in age and sex. We hypothesized that mindfulness practitioners would show fewer symptoms of distress, depression, anxiety, and stress than non-practitioners. Moreover, we hypothesized a protective effect of mindfulness practice in practitioners, assessed a second time at a three-week follow-up. We postulated that increased mindfulness practice during the COVID-19 pandemic would be associated with positive changes in symptoms of pandemic-related distress, depression, anxiety, and stress, particularly in experienced practitioners.

\section{Methods \\ Participants}

Participants (Table 1) were recruited on the social media platform WeChat in China. Practitioners (assessed on Feb $4-5 ; N=673$, and Feb 29-30; $N=521$ ) were directly recruited from a WeChat group of mindfulness practitioners practicing Pure Awareness mindfulness practices taught by the PARI (Pure Awareness Research Institute, see below). Non-practitioners (assessed on Feb $4-5 ; N=1550)$ were recruited from the general population and reached via a broad advertising campaign on WeChat targeting no particular demographics. Experience of mindfulness practice in the practitioner group ranged between six months and two years. Participants were excluded from further analyses if they spent less than five/more than $30 \mathrm{~min}$ to complete the required questionnaires. Although the two groups did not differ 
Table 1 Sample characteristics.

\begin{tabular}{|c|c|c|c|c|c|}
\hline & & $\begin{array}{l}\text { Non-practitioners } \\
\text { at peak time }\end{array}$ & $\begin{array}{l}\text { Practitioners at } \\
\text { peak time }\end{array}$ & $\begin{array}{l}\text { Practitioners at } \\
\text { three-week } \\
\text { assessment }\end{array}$ & $\begin{array}{l}\text { Practitioners at peak time } \\
\text { that were followed-up } \\
\text { three weeks later }\end{array}$ \\
\hline Total & & 1550 & 673 & 521 & 445 \\
\hline \multirow[t]{5}{*}{ Age } & $25-30$ & 218 & 34 & 28 & 22 \\
\hline & $31-40$ & 664 & 250 & 178 & 156 \\
\hline & $41-50$ & 500 & 284 & 229 & 196 \\
\hline & $51-60$ & 143 & 93 & 73 & 60 \\
\hline & $>60$ & 25 & 12 & 13 & 11 \\
\hline \multirow[t]{2}{*}{ Sex } & Female & 1040 & 477 & 394 & 333 \\
\hline & Male & 510 & 196 & 127 & 112 \\
\hline \multirow[t]{3}{*}{ Lockdown status } & $\begin{array}{l}\text { Complete (all the time } \\
\text { at home) }\end{array}$ & 1124 & 423 & 325 & 285 \\
\hline & $\begin{array}{l}\text { Partial (occasionally outside } \\
\text { for work) }\end{array}$ & 207 & 122 & 60 & 81 \\
\hline & None (working regularly) & 219 & 128 & 136 & 79 \\
\hline \multirow[t]{5}{*}{ Education } & $\begin{array}{l}\text { Junior high school education } \\
\text { and lower }\end{array}$ & 49 & 23 & 12 & 11 \\
\hline & $\begin{array}{l}\text { Senior high school and } \\
\text { equivalent }\end{array}$ & 155 & 42 & 34 & 28 \\
\hline & Vocational education & 324 & 115 & 88 & 71 \\
\hline & Undergraduate degree & 685 & 280 & 220 & 191 \\
\hline & Graduate degree & 337 & 213 & 167 & 144 \\
\hline \multirow[t]{3}{*}{ Location } & Hubei & 70 & 16 & 12 & 11 \\
\hline & $\begin{array}{l}\text { Beijing, Shanghai, Guangzhou, } \\
\text { Shenzhen }\end{array}$ & 704 & 305 & 169 & 208 \\
\hline & Other & 776 & 352 & 340 & 226 \\
\hline
\end{tabular}

significantly in gender (mean difference $=0.038$; $\mathrm{SE}=$ $0.021 ; 95 \% \mathrm{CI}=-0.004,0.080 ; \mathrm{t}(1317.255)=1.782 ; p=$ $0.075)$, there was a significant age difference for each of the four categorical groups $(25-30 \mathrm{y}$ : mean difference $=$ $0.090 ; \mathrm{SE}=0.012 ; 95 \% \mathrm{CI}=0.066,0.114 ; \mathrm{t}(1938.985)=$ $7.373 ; p<0.001 ; 31-40 \mathrm{y}$ : mean difference $=0.057 ; \mathrm{SE}=$ $0.057 ; 95 \% \mathrm{CI}=0.013,0.101 ; \mathrm{t}(1305.391)=2.531 ; p=$ $0.011 ; 41-50 \mathrm{y}:$ mean difference $=-0.099 ; \mathrm{SE}=0.022$; $95 \% \mathrm{CI}=-0.143, \quad-0.055 ; \mathrm{t}(1216.178)=-4.428 ; \quad p<$ $0.001 ; 51-60 \mathrm{y}:$ mean difference $=-0.046 ; 95 \% \mathrm{CI}=$ $-0.076,-0.016 ; \mathrm{t}(1100.141)=-3.020 ; p=0.003$; twotailed tests, equal variances not assumed). These two demographic variables were included as predictors in subsequent statistical analyses.

\section{Study design}

Pandemic-related distress, depression anxiety, and stress were assessed in non-practitioners $(N=1550)$ and practitioners $(N=673)$ at the peak of the COVID-19 pandemic in China (February 4-5, 2020). The same measures were assessed again at a three-week follow-up $(N=521$; February 29-30, 2020) in an overlapping sample of mindfulness practitioners $(N=445$ practitioners were assessed at both time points). All practitioners received instructions to practice meditation between the two assessment sessions and reported mindfulness practice frequency during the past two weeks. The study was approved by the local ethics committee (Fudan University). Informed consent was given at the beginning of the survey. Assessments were carried out via online questionnaires (SI-1 to SI-4). Distress was assessed using five questions related to the behavioral stress response to the COVID-19 pandemic (e.g., "I get nervous if someone nearby coughs or sneezes", 1 [not nervous] to 7 [very nervous] Likert scale). Stress, anxiety, and depression were assessed with the 22-item Impact of Event Scale-Revised 
$(\text { IES-R })^{45}$ questionnaire, the 7-item Generalized Anxiety Disorder-7 questionnaire (GAD-7) ${ }^{46}$, and the 20-item Center for Epidemiologic Studies Depression Scale (CESD) ${ }^{47}$, respectively, in Chinese ${ }^{48}$. The frequency of mindfulness practice was assessed with an 11-item questionnaire assessing the frequency of different mindfulness practices over the last two weeks (SI-5).

Practitioners were trained in mindfulness meditation by the PARI. Secular mindfulness practice as instructed by the PARI is designed to be applied in the workplace and domestic settings so as to be relevant to daily business and family life and does not include any spiritual or religious content. The training program practitioners underwent 6 to 24 months prior to the study teaches different exercises to increase mindfulness and reduce negatively valenced emotions like stress and anxiety. The practices trained are designed for autonomous practice after initial structured teaching courses (five 3-day courses to be taken over a 1-year period). The mindfulness practices trained here are mainly characterized by high degrees of meta-awareness and dereification in the phenomenological matrix of mindfulness practices proposed bz Lutz et al. ${ }^{49}$. Key features of the PARI mindfulness intervention are open-monitoring meditation, focused-attention meditation, and body scan meditation exercises. A specific exercise guide containing daily home mindfulness exercises for the period between February 8, 2020, and February 29, 2020, was developed and provided to the practitioners online. The guide included audio instructions for each exercise. Participants were asked to freely choose any exercises they wanted to practice. Based on former participation in structured courses, practitioners belonged to three subgroups. The courses taught increasingly advanced mindfulness meditation-related knowledge and skills and had to be taken in order; thus, practitioners that participated in beginner courses only were classified as beginners, and practitioners that took all courses were classified as advanced. All original information from both groups was anonymized with unique identification codes for further analyses before exporting the data from the online survey platform.

\section{Statistical analysis}

Statistical analyses were performed using IBM SPSS Statistics (Version 26). The total score of each questionnaire (SI-1 to SI-5) was normalized to 0.0-1.0 (i.e., expressed as a proportion of the maximum score of the respective scale), in order to achieve similar distributions for statistical analysis. Measures of mental health and Practice Frequency were continuous variables, whereas the variables Time, Group, Class, Sex, and Age were categorical. Outliers $(z$-score $>3$ or $<-3)$ were excluded before analysis. Since this was an observational study based on data collected during the height of the pandemic in China, no a priori power calculations could be performed. However, using G*Power we post-hoc computed the achieved statistical power ${ }^{50}$ for the ANOVAs and regression analyses. Because of the difference in sample sizes for the ANOVA of non-practitioners and practitioners at peak time, power calculations for the ANOVAs were conservatively based on a total sample size of $N=$ 1346 , twice the sample size of the smaller group. We used an alpha error probability of 0.05 .

\section{Group comparison}

First, in order to investigate potential differences in pandemic-related distress, depression, anxiety, and stress between mindfulness practitioners $(N=1550)$ and nonpractitioners $(N=673)$ at the peak of the pandemic, we conducted four ANOVAs, including the main effects Group (non-practitioner, beginner, intermediate, advanced), Age (25-30, 31-40, 41-50, 51-60, $\geq 61$ years) and Sex (male, female), as well as their two-way interactions in the model. We were mainly interested in the main effect of Group, as well as the interactions including the factor Group. Since exploratively including three-way interactions in the models did not result in any significant effects and did not improve model fit, assessed using adjusted $R^{2}$, three-way interactions were not included in the final models. For the four ANOVAs, Bonferroni correction was applied by adjusting the significance level for main effects and interactions: Statistical significance was accepted at $p<0.0125$. Pairwise comparisons were performed to follow-up significant main effects. Here, FDR adjustment was applied.

\section{Change within practitioners}

To probe whether the mental health of mindfulness practitioners changed during the initial phase of the COVID-19 pandemic, we compared pandemic-related distress, depression, anxiety, and stress at peak time $(N=$ $673)$ and at three-week follow-up $(N=521$; for $N=445$ practitioners data were available at both time points) using hierarchical linear models (HLMs). We included the categorical variables Time (peak, three-week follow-up), Experience (beginner, intermediate, advanced), Age (25-30, 31-40, 41-50, 51-60, $\geq 61$ years), and Sex (male, female) and the continuous variable Practice Frequency as main effects in the model, as well as all two-way interactions. A diagonal covariance structure was selected for the repeated measures. Since adding the respective three-, four-way, and five-way interactions did not improve model fit, as assessed with Akaike information criteria, and did not result in significant effects, these were not retained in the final model. We were primarily interested in significant main effects or interactions of Time, Practice Frequency, and Experience. Bonferroni correction was applied to correct for running four HLMs by adjusting the 


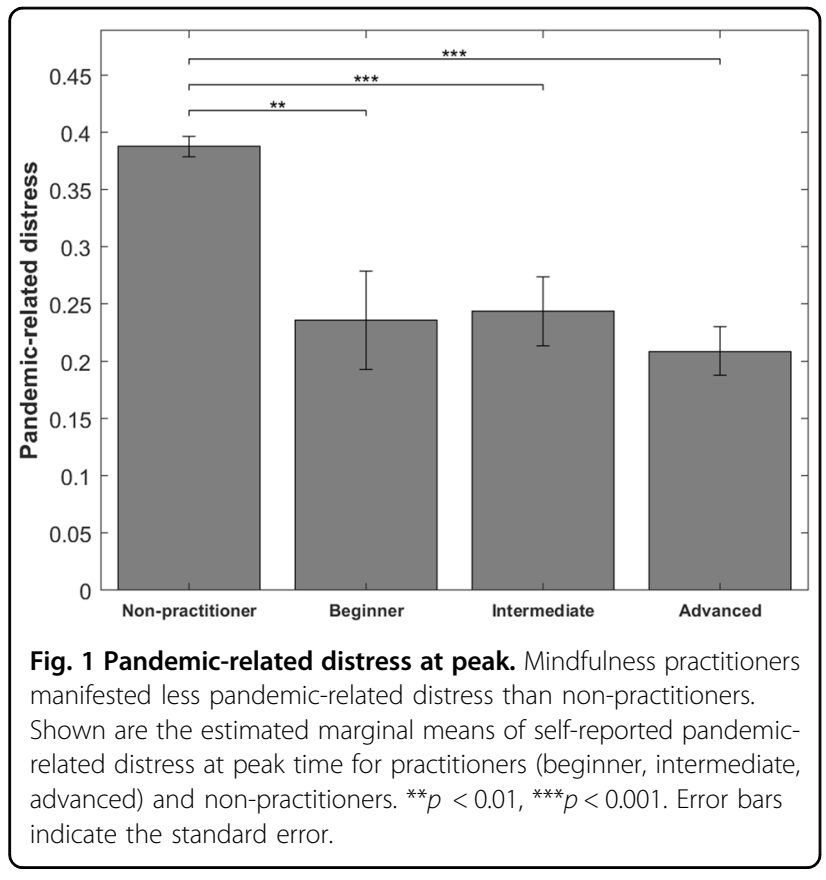

significance level for main effects and interactions: Statistical significance was accepted at $p<0.0125$. Pairwise comparisons were performed to follow-up the significant interaction and main effects. For pairwise comparisons, FDR adjustment was applied.

\section{Practice effect}

To test our main hypothesis of potential mindfulness practice effects on mental health during the pandemic, change in individual symptom scores of mindfulness practitioners $(N=445)$ was regressed on Practice Frequency during the last two weeks while controlling for Age (25-30, 31-40, 41-50, 51-60, $\geq 61$ years), Sex (male, female) and baseline symptoms. The age categories were recoded into four dummy variables. The change was computed as the score of distress/depression/anxiety/ stress at peak time minus the same score at three-week follow-up, for each practitioner. Thus, positive changes indicated improvement. We hypothesized a doseresponse relationship between Practice Frequency and improvement in measures of mental health during the COVID-19 pandemic. Bonferroni correction was applied by adjusting the threshold for accepting statistical significance for the overall models and the individual regression coefficients to $p<0.0125$. The regression analyses were then repeated after splitting the data into beginner, intermediate, and advanced practitioner subgroups. Here, FDR adjustment (12 follow-up regression analyses) was performed (for both the significance of the models and the regression coefficients). Finally, to assess the relationship between practice frequency and symptoms at baseline, we calculated the Pearson correlation coefficient.

\section{Results \\ Group comparison}

First, we compared mental health between mindfulness practitioners and non-practitioners at the peak of the COVID-19 outbreak in China, running four separate ANOVAs (supplementary table 1, supplementary table 2 for descriptive statistics). There was a significant main effect of Group for scores of pandemic-related distress $\left(\mathrm{F}(3,2195)=27.487 ; \quad p<0.001 ; \quad \eta_{\mathrm{p}}{ }^{2}=0.036 ; \quad\right.$ achieved power $=1$; Fig. 1). Pairwise comparisons indicated that non-practitioners differed significantly from practitioners at beginner (mean difference $=.151 ; \mathrm{SE}=0.044 ; \mathrm{t}(1796)=$ 3.432; $95 \% \mathrm{CI}=0.065,0.238 ; p_{\text {FDR-adjusted }}=0.001$; mean difference in raw scores $=4.53$ ), intermediate (mean difference $=0.144 ; \mathrm{SE}=0.031 ; \mathrm{t}(1690)=4.645 ; 95 \% \mathrm{CI}=$ $0.082,0.205 ; p_{\text {FDR-adjusted }}<0.001$; mean difference in raw scores $=4.32$ ) and advanced (mean difference $=0.179$; $\mathrm{SE}=0.023 ; \mathrm{t}(1831)=7.783 ; 95 \% \mathrm{CI}=0.135,0.223 ; p_{\text {FDR- }}$ adjusted $<0.001$; mean difference in raw scores $=5.37$ ) experience levels, reporting less pandemic-related distress. There were no significant differences between subgroups of practitioners and no significant effects of Group for scores of depression, anxiety, and stress. However, the main effects of Age for scores of depression $(\mathrm{F}(4,2195)=$ 5.912; $p<0.001 ; \quad \eta_{\mathrm{p}}^{2}=0.011 ;$ achieved power $\left.=0.867\right)$ and anxiety $\left(\mathrm{F}(4,2195)=6.455 ; p<0.001 ; \eta_{\mathrm{p}}{ }^{2}=0.012\right.$; achieved power $=0.898$; Supplementary Fig. 1) were significant. Regarding depressive symptoms, pairwise comparisons showed that subjects aged 25-30y reported significantly more symptoms than subjects aged $41-50$ y (mean difference $=0.044 ; \mathrm{SE}=0.019 ; \mathrm{t}(1834)=$ $2.316 ; 95 \% \mathrm{CI}=0.006,0.081 ; p_{\text {FDR-adjusted }}=0.043$; mean difference in raw scores $=2.64$ ) and $>60 \mathrm{y}$ (mean difference $=0.106 ; \mathrm{SE}=0.042 ; \mathrm{t}(387)=2.524 ; 95 \% \mathrm{CI}=0.023$, $0.188 ; p_{\text {FDR-adjusted }}=0.031$; mean difference in raw scores $=6.36$ ). Likewise, subjects aged $31-40 \mathrm{y}$ also showed significantly more symptoms than older subjects aged $41-50 \mathrm{y}$ (mean difference $=0.036 ; \mathrm{SE}=0.009 ; \mathrm{t}$ $(2247)=4.0 ; 95 \% \mathrm{CI}=0.018,0.054 ; p_{\text {FDR-adjusted }}=0.001$; mean difference in raw scores $=2.16$ ), $51-60 \mathrm{y}$ (mean difference $=0.035 ; \mathrm{SE}=0.014 ; \mathrm{t}(1513)=2.5 ; 95 \% \mathrm{CI}=$ $0.008,0.062 ; p_{\text {FDR-adjusted }}=0.037$; mean difference in raw scores $=2.10)$ and $>60 \mathrm{y}$ (mean difference $=0.098 ; \mathrm{SE}=$ $0.031 ; \mathrm{t}(1250)=2.513 ; 95 \% \mathrm{CI}=0.022,0.174 ; p_{\text {FDR-adjusted }}$ $=0.031$; mean difference in raw scores $=5.88$ ). Similarly, with regard to anxiety scores, pairwise comparisons showed that participants aged 25-30y reported significantly more anxiety symptoms than subjects over $60 \mathrm{y}$ (mean difference $=0.119 ; \mathrm{SE}=0.049 ; \mathrm{t}(387)=2.429 ; 95 \%$ $\mathrm{CI}=0.022,0.215 ; p_{\mathrm{FDR} \text {-adjusted }}=0.041$; mean difference in raw scores $=3.33$ ), and subjects aged $31-40 \mathrm{y}$ also 
manifested more symptoms than subjects aged $41-50 \mathrm{y}$ (mean difference $=0.045 ; \mathrm{SE}=0.011 ; \mathrm{t}(2247)=4.091$; $95 \% \mathrm{CI}=0.023,0.066 ; p_{\text {FDR-adjusted }}<0.001$; mean difference in raw scores $=1.26), 51-60 \mathrm{y}$ (mean difference $=$ 0.049; $\mathrm{SE}=0.016 ; \mathrm{t}(1513)=3.063 ; 95 \% \mathrm{CI}=0.018,0.081$; $p_{\text {FDR-adjusted }}=0.010$; mean difference in raw scores $=1.37$ ) and $>60 \mathrm{y} \quad($ mean difference $=0.121 ; \quad \mathrm{SE}=0.045$; $\mathrm{t}(1250)=2.689 ; 95 \% \mathrm{CI}=0.032,0.210 ; p_{\text {FDR-adjusted }}=$ 0.026 ; mean difference in raw scores $=3.39$ ). Thus, younger participants below the age of 40 reported more symptoms of depression and anxiety than older participants. No other main effects or interactions were significant at the Bonferroni corrected significance threshold of $p<0.0125$.

\section{Change in practitioners}

To investigate changes in the mental health status of mindfulness practitioners during the COVID-19 pandemic in China, we performed hierarchical linear modeling for scores of pandemic-related distress, depression, anxiety, and stress (supplementary table 3, supplementary table 2 for descriptive statistics). For pandemic-related distress, we found a significant interaction of Time $\times$ Age $(\mathrm{F}(4$, $546.106=4.007 ; p=0.003$; Fig. 2). Pairwise comparisons showed that pandemic-related distress decreased from peak to follow-up in younger practitioners. Specifically, pandemic-related distress decreased significantly in the age groups $25-30 \mathrm{y}$ (mean difference $=0.130 ; \mathrm{SE}=0.031 ; \mathrm{t}$ $(595.079)=4.194 ; 95 \% \mathrm{CI}=0.069,0.190 ; p_{\text {FDR-adjusted }}<$ 0.001 ; mean difference in raw scores $=3.90), 31-40 \mathrm{y}$ $($ mean difference $=0.0 .095 ; \mathrm{SE}=0.013 ; \mathrm{t}(579.642)=$ 7.308; $95 \% \mathrm{CI}=0.070,0.120 ; p_{\mathrm{FDR}-\text { adjusted }}<0.001$; mean

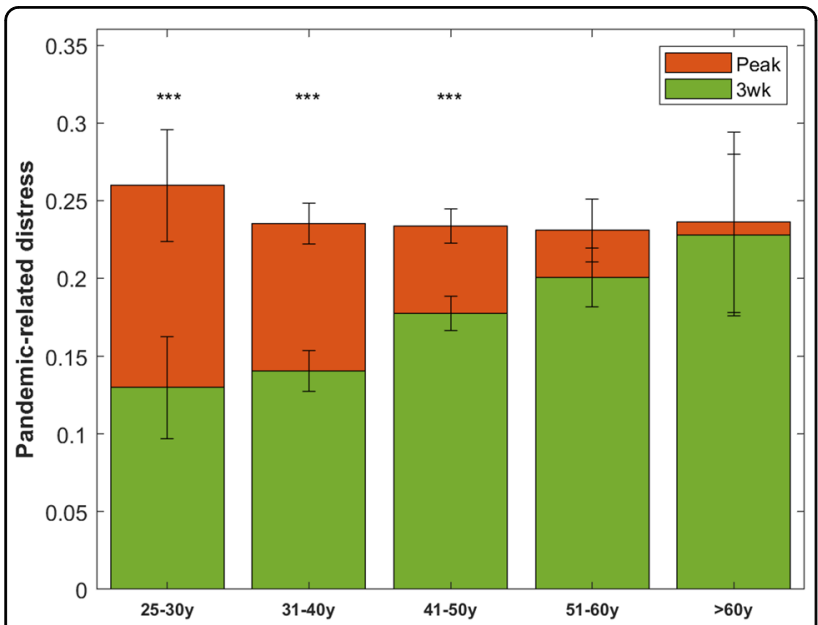

Fig. 2 Age-dependent decrease of pandemic-related distress. Pandemic-related distress decreased from peak to follow-up in younger mindfulness practitioners. Shown are the estimated marginal means of self-reported pandemic-related distress in practitioners at peak and three-week follow-up in the different age groups. ${ }^{* *} p<$ 0.001. Error bars indicate the standard error. $y=$ years. difference in raw scores $=2.85$ ) and $41-50 \mathrm{y}$ (mean difference $=0.056 ; \mathrm{SE}=0.011 ; \mathrm{t}(554.951)=5.091 ; 95 \% \mathrm{CI}=$ $0.034,0.078 ; p_{\text {FDR-adjusted }}<0.001$; mean difference in raw scores $=1.68$ ).

For depressive symptoms, we found a significant main effect of Practice Frequency $(\mathrm{F}(1,1060.453)=12.527 ; p<$ 0.001). There was a significant negative Pearson correlation between Practice Frequency and depressive symptoms $(r=-0.286, p<0.001)$, the more frequent practice was associated with lower reported symptoms. Furthermore, we found a significant main effect of Time (F $(1$, $546.766)=20.353 ; \quad p<0.001), \quad$ due to practitioners reporting more depressive symptoms at follow-up (mean difference $=-0.042 ; \quad \mathrm{SE}=0.010 ; \quad \mathrm{t}(488.304)=-4.372$; $95 \% \mathrm{CI}=-0.061,-0.023 ; p_{\mathrm{FDR} \text {-adjusted }}<0.001$; mean difference in raw scores $=-2.52$ ).

For symptoms of anxiety, we again found a significant main effect of Practice Frequency $(F(1,921.902)=15.103$; $p<0.001)$. There was a significant negative Pearson correlation between practice frequency and anxiety symptoms $(r=-0.263, p<0.001)$. We also found a significant main effect of Age $(\mathrm{F}(4,948.160)=3.370 ; p=0.009)$. Younger practitioners aged 25-30y showed significantly more symptoms of anxiety than practitioners aged $41-50 \mathrm{y}$ (mean difference $=0.076 ; \mathrm{SE}=0.026 ; \mathrm{t}(663.157)=2.923$; $95 \% \mathrm{CI}=0.025,0.127 ; p_{\mathrm{FDR}-\text { adjusted }}=0.018$; mean difference in raw scores $=2.13)$ and $51-60 \mathrm{y}$ (mean difference $=$ $0.074 ; \quad \mathrm{SE}=0.028 ; \mathrm{t}(678.537)=2.643 ; \quad 95 \% \mathrm{CI}=0.018$, $0.130 ; p_{\text {FDR-adjusted }}=0.033$; mean difference in raw scores $=$ 2.07). Similarly, practitioners aged $31-40 y$ showed more anxiety than practitioners in the age group $41-50 \mathrm{y}$ (mean difference $=0.038 ; \quad \mathrm{SE}=0.012 ; \quad \mathrm{t}(746.099)=3.167 ; \quad 95 \%$ $\mathrm{CI}=0.016,0.061 ; p_{\text {FDR-adjusted }}=0.009$; mean difference in raw scores $=1.06$ ).

No other main effects or interactions resulted in significant effects at the Bonferroni corrected significance threshold of $p<0.0125$.

\section{Practice effect}

To further test whether individual changes in symptom scores were related to practice frequency, the change in symptom scores (available at both time points for $N=445$ practitioners) was regressed on practice frequency during the last two weeks (supplementary table 4). Practice Frequency significantly predicted improvement for symptoms of depression (ANOVA: $\mathrm{F}(7437)=15.586 ; p<0.001 ; R^{2}=$ 0.200; Coefficient: $B=0.108 ; \mathrm{SE}_{\mathrm{B}}=0.022 ; \mathrm{CI}=0.066,0.151$; $\beta=0.225 ; \mathrm{t}(437)=5.003 ; p<0.001 ; p R^{2}=0.054 ;$ achieved power $=0.999$ ), anxiety (ANOVA: $\mathrm{F}(7,437)=40.825 ; p<$ 0.001; $R^{2}=0.395$; Coefficient: $B=0.095 ; \mathrm{SE}_{\mathrm{B}}=0.022 ; \mathrm{CI}=$ $0.052,0.137 ; \beta=0.171 ; \mathrm{t}(437)=4.374 ; p<0.001 ; p R^{2}=$ 0.042 ; achieved power $=0.993$ ) and stress (ANOVA: $\mathrm{F}(7437)=18.024 ; \quad p<0.001 ; \quad R^{2}=0.224 ; \quad$ Coefficient: $B=0.061 ; \quad \mathrm{SE}_{\mathrm{B}}=0.019 ; \quad \mathrm{CI}=0.023, \quad 0.098 ; \quad \beta=0.139 ;$ 


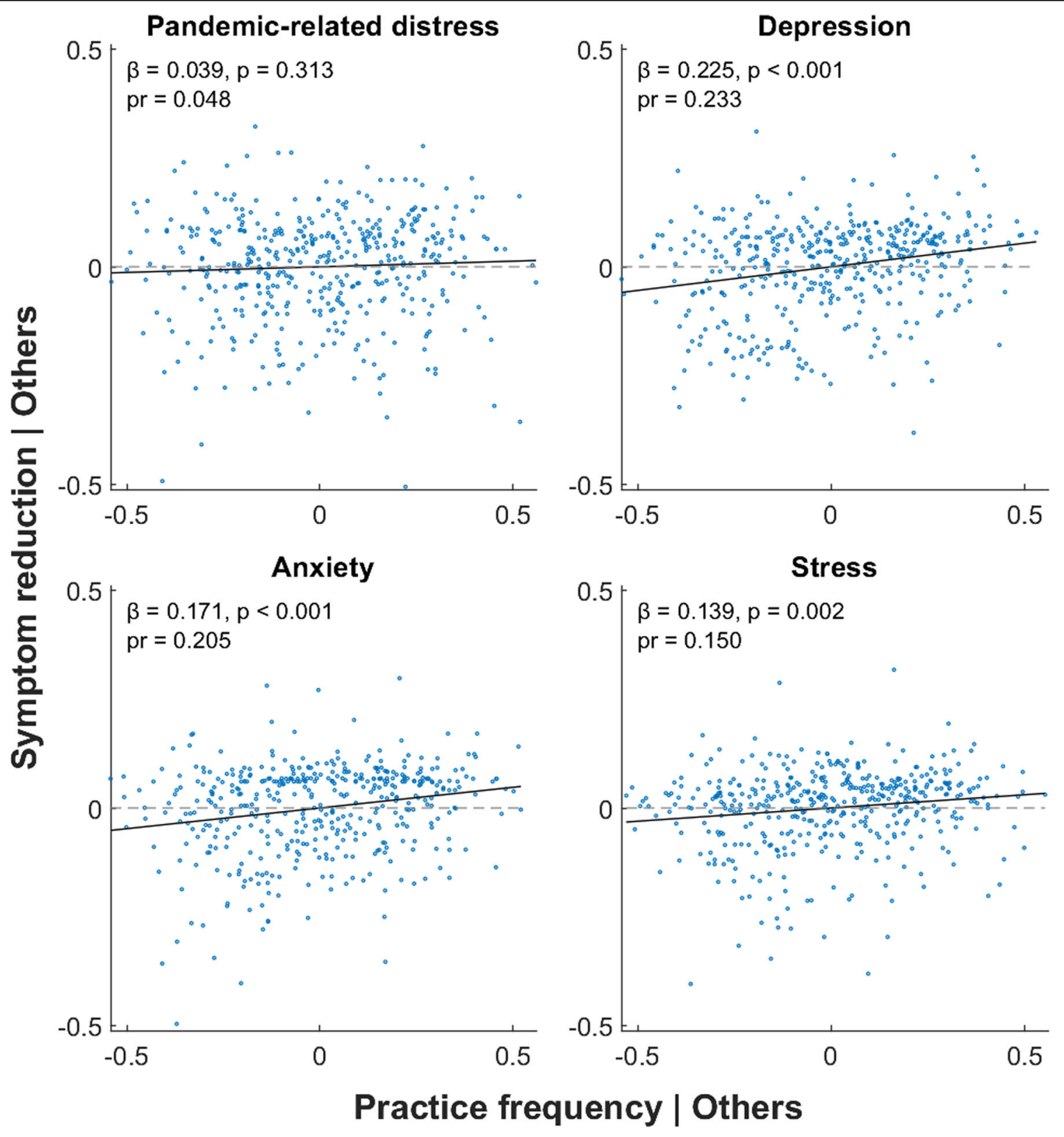

Fig. 3 Practice-dependent symptom improvement. Partial regression plots showing the improvement in self-reported symptoms of depression (normalized CES-D score at peak - normalized CES-D score at three-week follow-up), anxiety (normalized GAD-7 score at peak - normalized GAD-7 score at three-week follow-up), and stress (normalized IES-R score at peak - normalized IES-R score at three-week follow-up) with increased frequency of mindfulness practice when controlling for other variables (age, sex, and symptoms at baseline). $\beta=$ beta coefficient, pr $=$ partial correlation.

$\mathrm{t}(437)=3.176 ; p=0.002 ; p R^{2}=0.023 ;$ achieved power $=$ 0.892) when including Age, Sex and the respective baseline scores of depression/anxiety/stress as control variables; the more frequent practice was associated with symptom reduction (Fig. 3). For distress, we did not find an effect of Practice Frequency. Looking at practitioners with different levels of mindfulness practice experience (supplementary table 5), in advanced practitioners, the effect of Practice
Frequency was significant for the improvement of depressive symptoms (ANOVA: $\mathrm{F}(7202)=11.050 ; p_{\text {FDR-adjusted }}<0.001$; $R^{2}=0.277$; Coefficient: $B=0.105 ; \mathrm{SE}_{\mathrm{B}}=0.032 ; \mathrm{CI}=0.043$, $0.168 ; \beta=0.211 ; \mathrm{t}(202)=3.316 ; p_{\text {FDR-adjusted }}=0.006 ; p R^{2}=$ 0.052 ) and improvement of anxiety symptoms (ANOVA: $F$ $(7202)=16.763 ; p_{\text {FDR-adjusted }}<0.001 ; R^{2}=0.367$; Coefficient: $B=0.108 ; \mathrm{SE}_{\mathrm{B}}=0.030 ; 95 \% \mathrm{CI}=0.049,0.168 ; \beta=0.211$; $\left.\mathrm{t}(202)=3.596 ; \quad p_{\text {FDR-adjusted }}=0.005 ; \quad p R^{2}=0.060\right) ; \quad$ more 
practice was associated with symptom reduction. For depression, the effect of Practice Frequency was also significant in intermediate practitioners (ANOVA: $\mathrm{F}(7,81)=$ $4.721 ; \quad p_{\text {FDR-adjusted }}<0.001 ; \quad R^{2}=0.290 ;$ Coefficient: $B=$ $0.122 ; \quad \mathrm{SE}_{\mathrm{B}}=0.045 ; \quad 95 \% \quad \mathrm{CI}=0.033, \quad 0.212 ; \quad \beta=0.272$; $\left.\mathrm{t}(81)=2.724 ; p_{\text {FDR-adjusted }}=0.032 ; p R^{2}=0.084\right)$.

Regarding the relationship between Practice Frequency and baseline symptoms, we found significant negative associations between Practice Frequency and baseline scores of pandemic-related distress $(r=-0.189 ; p<$ $0.001)$, depression $(r=-0.239 ; \quad p<0.001)$, anxiety $(-0.259 ; p<0.001)$ and stress $(r=-0.197 ; p<0.001)$.

\section{Discussion}

Since emerging evidence suggests that the COVID-19 pandemic and the counter-measures it engendered have a considerable negative impact on mental health ${ }^{12,15-18}$, it is crucial to identify scalable, low-cost interventions that can safeguard public mental health during this and future pandemic crises $^{9,25}$. In our observational study, we investigated the relationship of mindfulness practice and pandemic-related distress, depressive symptoms, anxiety symptoms, and stress, in a large group of mindfulness practitioners, surveyed at the peak of new COVID-19 infections in China and three weeks later. Symptom scores of practitioners at peak time were also compared to a group of non-practitioners, surveyed at peak time only.

\section{Group comparison}

The level of pandemic-related distress reported at peak time was significantly lower in practitioners, compared to non-practitioners, when controlling for possible confounding effects of age and sex. We found no differences in symptoms of depression, anxiety, and stress between both groups. These findings might be explained by the fact that pandemic-related distress could be more sensitive to specific emotional/behavioral pandemic-related alterations (e.g., getting nervous if someone nearby coughs or sneezes). Such symptoms might be more amenable to change (even after limited amounts of mindfulness practice) than clinical and more trait-like characteristics like symptoms of depression, anxiety, or stress. We also found that older participants manifested generally fewer symptoms of depression and anxiety, compared to younger participants, regardless of group. This finding agrees with former studies reporting agerelated effects for depression and anxiety ${ }^{50,51}$.

\section{Change in practitioners}

In line with our hypothesis of protective psychological effects of mindfulness practice during the COVID-19 pandemic, we found a reduction in pandemic-related distress at follow-up in younger practitioners. We did not find significant differences in anxiety or stress between the peak and three-week follow-up surveys. It is possible that a putative negative psychological impact of the pandemic has been protected against by a positive influence of mindfulness meditation practice. In support of this interpretation, considerable evidence has demonstrated that mindfulness meditation may reduce anxiety, stress, and depressive symptoms and that the COVID-19 pandemic has generally increased such symptoms ${ }^{9}$. Indeed, practice frequency correlated negatively with symptoms of depression and anxiety. However, we also found an increase in depressive symptoms from peak to follow-up. This increase could-in part-be due to the strict quarantine measures during the time of this study ${ }^{14,19}$.

\section{Practice effect}

The regression of symptom improvement on practice frequency before follow-up showed a dose-dependent reduction in symptoms of anxiety, depression, and stress. Practitioners who practiced more frequently during the critical phase of the COVID-19 pandemic in China had a better mental health status at follow-up than those practicing less frequently. This association was most robust for depression (partial correlation squared $=0.054$ ). Analyzing this association in each of the three subgroups separately, we found the strongest association in advanced practitioners (for both depression and anxiety). Thus, the effectiveness of autonomous mindfulness practice may depend critically on an appropriate amount of previous structured training. Interestingly, even though more frequent mindfulness practice was associated with fewer symptoms of depression, anxiety, and stress in practitioners, practitioners did not differ significantly from nonpractitioners on these variables at peak. The relative weakness of the associations between practice frequency and symptom improvement might explain why the between-group comparison did not result in significant effects. Conversely, despite the group difference regarding pandemic-related distress, we did not find an association between practice frequency and pandemic-related distress in practitioners. The group difference in pandemic-related distress might thus be due to other factors independent of mindfulness practice frequency that could be related to mindfulness practice (e.g., better emotional control resulting from long-term practice) but could also be independent of mindfulness practice altogether (i.e., confounding socio-demographic variables).

\section{Comparison with other findings and potential mechanisms of mindfulness practice}

Regular mindfulness practice is accompanied by structural and functional changes in brain regions involved in the regulation of emotion, attention, and selfawareness $^{43,52}$. Consequently, mindfulness-based interventions have been increasingly studied as a treatment 
tool for psychiatric conditions such as depression and anxiety ${ }^{27,29,53}$. Our findings are in line with previous meta-analyses showing that mindfulness-based interventions are useful treatments for reducing distress, anxiety, depression, and stress ${ }^{29}$ that are not only effective in clinical populations but also improve mental health in healthy individuals ${ }^{37}$. The dose-dependent reduction in symptoms of depression, anxiety, and stress concords well with former studies demonstrating that mindfulness practice can improve stress resilience ${ }^{35,54}$ and results in an enhanced ability to find meaning in adverse events ${ }^{55}$, like the COVID-19 pandemic.

Emotion regulation is known to be dysfunctional across many mental disorders ${ }^{56,57}$, such as depression ${ }^{58}$. Dysfunctional emotion regulation during a time of crisis will likely have a particularly detrimental effect on one's mental health status. Mindfulness practice strengthens the ability to consciously notice emotional states and improves their regulation ${ }^{43,59-62}$. The emotion regulation strategies relevant for mindfulness practice can be differentiated into top-down strategies (e.g., affect labeling) and bottom-up strategies (e.g., sensory-perception) ${ }^{63}$. It has been suggested that top-down emotion regulation strategies may be more relevant in short-term mindfulness practitioners, while bottom-up strategies could play a greater role in long-term practitioners ${ }^{64}$, such as the advanced practitioners in our study. Since emotional self-regulation is at the core of resilience, an increase in resilience due to mindfulness practice could protect against stress and anxiety during the pandemic. Increases in stress resilience due to mindfulness training have also been reported for other contexts with high levels of stress ${ }^{38-40}$. Recently, resilience has been shown to protect against COVID-19 related distress and was linked to lower rates of anxiety and depression ${ }^{11}$. Further research should investigate the relationship between mindfulness practice, emotion regulation, resilience, and mental health status.

Besides, other mechanisms play important roles in the effects of mindfulness-based interventions. A systematic review of mindfulness-based cognitive therapy (MBCT) in the treatment of recurrent major depressive disorder found that alterations in mindfulness, ruminations, worries, and meta-awareness were associated with, predicted, or mediated the effects of MBCT interventions ${ }^{65}$. In particular, ruminations-excessive, repetitive, and uncontrolled negatively valenced thoughts-are likely to be increased during a time of crisis like the COVID-19 pandemic and have been shown to decrease under MBCT in a randomized controlled trial ${ }^{66}$. Reductions in ruminations, worrying, and unconstructive repetitive thoughts in general ${ }^{67}$ due to mindfulness meditation training may partly explain the reductions in scores of depression, anxiety, and stress in this study. A decrease in mindwandering and higher meta-awareness of distraction ${ }^{68}$ and non-specific aspects of mindfulness practice such as increased self-efficacy may also play a role. Our findings highlight that an appropriate amount of guided mindfulness training is crucial for self-administrated mindfulness practice to be effective under real-life conditions. Differential effects of different levels of mindfulness training experience have also been investigated in recent fMRI studies. For example, the total amount of retreat meditation practice correlated with a reduction of right amygdala activation for negative pictures in experienced practitioners having accumulated about $9000 \mathrm{~h}$ of lifetime meditation practice, while no reduction in amygdala sensitivity to negative stimuli was seen after a single 8week mindfulness-based stress reduction (MBSR) program $^{69}$. In contrast, another neuroimaging study found that changes in hippocampal-cortical connectivity occurred even after a single 8-week MBSR intervention ${ }^{35}$. These investigations illustrate that changes in brain network activity underlying beneficial effects of mindfulness practice accrue over time in a complex manner, in agreement with greater benefit in advanced practitioners in our study. Unfortunately, subjects participating in MBSR/MBCT interventions often do not carry out the recommended amount of home practice ${ }^{70}$. In our investigation, practitioners received web-based instructions to encourage mindfulness training during the pandemic. In the future, internet-based and smartphone-based mindfulness interventions could be a low-cost option to make mindfulness-based treatment available to large populations $^{9,71}$. This could also overcome the challenge of appropriately training mindfulness meditation teachers, identified as one of the barriers hindering the translation of mindfulness research into clinical practice ${ }^{72}$.

\section{Limitations}

Causal interpretations of our data are limited by the observational nature of our study. Selection bias may have contributed to the difference between non-practitioners and practitioners. It is possible that practitioners and nonpractitioners differed in unmeasured characteristics such as environmental and socioeconomic factors or personality/cognitive styles, which might explain why practitioners experienced less pandemic-related distress. Furthermore, the interpretability of the comparison between measures at peak time and three-week follow-up in practitioners would have been improved if the sample of non-practitioners could have been followed-up for a second assessment as well since mental states might have worsened during the pandemic in non-practitioners. Finally, it needs to be noted that the magnitude of mindfulness practice effects in this study is relatively small. However, it is encouraging that a positive association between frequency of practice and improvement in symptoms of depression, anxiety, and stress could be 
found, given that we looked at only three weeks of unsupervised, autonomous practice (thus, one expects a considerably smaller effect than for classic 8-week MBSR programs involving considerable didactic instruction, frequent group sessions and a daylong retreat) ${ }^{27}$. Further experimental studies should corroborate our findings and elucidate whether more prolonged or more intensive mindfulness practice may elicit stronger protective effects during the pandemic.

\section{Conclusion}

Our observational study demonstrates that practitioners of mindfulness meditation manifested less pandemicrelated distress than non-practitioners during the COVID-19 pandemic in China. Importantly, practice frequency was associated with improvements in symptoms of depression, anxiety, and stress, especially in experienced practitioners. Further interventional studies should corroborate our results. If implemented effectively, mindfulnessbased interventions might be a low-cost option to safeguard public mental health at times of crisis like the current COVID-19 pandemic.

\section{Acknowledgements}

We would like to thank Jingzhu Liu, Hongyu Zhang, Wenjiang Fu, Yan Li, Zhang Zhao, and Lei Shen for their valuable support in this project. We would like to acknowledge the support from the 111 Project (No. B18015), the key project of Shanghai Science and Technology (No. 16JC1420402), Shanghai Municipal Science and Technology Major Project (No. 2018SHZDZX01), National Key R\&D Program of China (No. 2018YFC1312900), and National Natural Science Foundation of China (NSFC 91630314).

\section{Author details \\ ${ }^{1}$ Fanhai International School of Finance, Fudan University, Shanghai, China. ${ }^{2}$ Institute of Science and Technology for Brain-Inspired Intelligence, Fudan University, Shanghai, China. ${ }^{3}$ School of Economics, Fudan University, Shanghai, China. ${ }^{4}$ Pure Awareness Research Institute, Shanghai, China. ${ }^{5}$ Behavioural and Clinical Neurosciences Institute, Department of Psychiatry, University of Cambridge, Cambridge, United Kingdom}

\section{Conflict of interest}

The PARI is an organization with commercial interests. M.Y.C., J.Y., and H.Z. are teachers of PARI mindfulness courses and beneficiaries of revenues generated through these courses. The special three-week exercise program for mindfulness practice during the pandemic, designed for this study, was provided for free by the PARI. J.Y. and H.Z. were involved in recruiting participants, implementing and disseminating the online questionnaires, and assisting with preprocessing the data. The PARI was initiated at the end of 2019 with the aim of conducting translational research on mindfulness in a real-life setting. PARI did not contribute any funding or other resources except for the above-mentioned free courses and the practical involvement in data collection. Apart from initial data preprocessing, the PARI was not involved in data analysis. The remaining authors declare no competing interests.

\section{Publisher's note}

Springer Nature remains neutral with regard to jurisdictional claims in published maps and institutional affiliations.

Supplementary information The online version contains supplementary material available at https://doi.org/10.1038/s41398-021-01459-8.
Received: 4 July 2020 Revised: 9 May 2021 Accepted: 18 May 2021

Published online: 28 May 2021

\section{References}

1. Depression and other common mental disorders: global health estimates (World Health Organization, Geneva, 2017).

2. Kessler, R. C. The effects of stressful life events on depression. Annu. Rev. Psychol. 48, 191-214 (1997).

3. Slavich, G. M. \& Irwin, M. R. From stress to inflammation and major depressive disorder: a social signal transduction theory of depression. Psychol. Bull. 140 774-815 (2014).

4. Ge, L., Yap, C. W., Ong, R. \& Heng, B. H. Social isolation, loneliness and their relationships with depressive symptoms: a population-based study. PLOS ONE 12, e0182145 (2017).

5. Matthews, T. et al. Social isolation, loneliness and depression in young adulthood: a behavioural genetic analysis. Soc. Psychiatry Psychiatr. Epidemiol. 51, 339-348 (2016).

6. Santini, Z. I. et al. Social disconnectedness, perceived isolation, and symptoms of depression and anxiety among older Americans (NSHAP): a longitudinal mediation analysis. Lancet Public Health 5, e62-e70 (2020).

7. Bonde, J. P. et al. Risk of depressive disorder following disasters and military deployment: systematic review with meta-analysis. Br. J. Psychiatry 208, 330-336 (2016).

8. Stoddard F. J., Pandya A. \& Katz C. L. Disaster Psychiaty: Readiness, Evaluation, and Treatment (American Psychiatric Publishing Inc., 2012).

9. Vatansever D., Wang S. \& Sahakian B. J. Covid-19 and promising solutions to combat symptoms of stress, anxiety and depression. Neuropsychopharmacology https://doi.org/10.1038/s41386-020-00791-9 (2020).

10. Xiang, Y.-T. et al. Timely mental health care for the 2019 novel coronavirus outbreak is urgently needed. Lancet Psychiatry 7, 228-229 (2020).

11. Barzilay, R. et al. Resilience, COVID-19-related stress, anxiety and depression during the pandemic in a large population enriched for healthcare providers. Transl. Psychiatry 10, 291 (2020).

12. Mertens, G., Gerritsen, L., Duijndam, S., Salemink, E. \& Engelhard, I. M. Fear of the coronavirus (COVID-19): Predictors in an online study conducted in March 2020. J. Anxiety Disord. 74, 102258 (2020).

13. Asmundson, G. J. G. \& Taylor, S. Coronaphobia: fear and the 2019-nCoV outbreak. J. Anxiety Disord. 70, 102196 (2020).

14. Brooks, S. K. et al. The psychological impact of quarantine and how to reduce it: rapid review of the evidence. Lancet 395, 912-920 (2020).

15. Wang C. et al. Immediate Psychological Responses and Associated Factors during the Initial Stage of the 2019 Coronavirus Disease (COVID-19) Epidemic among the General Population in China. Int. J. Environ. Res. Public Health $\mathbf{1 7}$ 1729 (2020).

16. Qiu, J. et al. A nationwide survey of psychological distress among Chinese people in the COVID-19 epidemic: implications and policy recommendations. Gen. Psychiatr. 33, e100213 (2020).

17. Afshar Jahanshahi, A., Mokhtari Dinani, M., Nazarian Madavani, A., Li, J. \& Zhang S. X. The distress of Iranian adults during the Covid-19 pandemic-More distressed than the Chinese and with different predictors. Brain Behav. Immun. 87, 124-125 (2020).

18. Rossi, R. et al. COVID-19 pandemic and lockdown measures impact on mental health among the general population in Italy. Front. Psychiatry 11, 790 (2020).

19. Meda, N. et al. Students' mental health problems before, during, and after COVID-19 lockdown in Italy. J. Psychiatr. Res. 134, 69-77 (2021).

20. Bell, V. \& Wade, D. Mental health of clinical staff working in high-risk epidemic and pandemic health emergencies a rapid review of the evidence and living meta-analysis. Soc. Psychiatry Psychiatr. Epidemiol. 56, 1-11 (2020).

21. Rossi, R. et al. Mental health outcomes among frontline and second-line health care workers during the Coronavirus Disease 2019 (COVID-19) Pandemic in Italy. JAMA Netw. Open 3, e2010185 (2020).

22. Lai, J. et al. Factors associated with mental health outcomes among health care workers exposed to Coronavirus Disease 2019. JAMA Netw. Open 3, e203976 (2020).

23. Rohde, C., Hougaard Jefsen, O., Noerremark, B., Aalkjaer Danielsen, A. \& Dinesen Østergaard, S. Psychiatric symptoms related to the COVID-19 pandemic. Acta Neuropsychiatr. 32, 274-276 (2020).

24. Duan, L. \& Zhu, G. Psychological interventions for people affected by the COVID-19 epidemic. Lancet Psychiatry 7, 300-302 (2020). 
25. Holmes, E. A. et al. Multidisciplinary research priorities for the COVID-19 pandemic: a call for action for mental health science. Lancet Psychiatry https://doi. org/10.1016/S2215-0366(20)30168-1 (2020).

26. Moreira, P. S. et al. Protective elements of mental health status during the COVID-19 outbreak in the Portuguese population. Int. J. Environ. Res. Public Health 18, 1910 (2021).

27. Wielgosz, J., Goldberg, S. B., Kral, T. R. A., Dunne, J. D. \& Davidson, R. J. Mindfulness meditation and psychopathology. Annu Rev. Clin. Psychol. 15, 285-316 (2019).

28. Kabat-Zinn, J. Some reflections on the origins of MBSR, skillful means, and the trouble with maps. Contemp. Buddhism 12, 281-306 (2011).

29. Khoury, B. et al. Mindfulness-based therapy: a comprehensive meta-analysis. Clin. Psychol. Rev. 33, 763-771 (2013).

30. Hofmann, S. G., Sawyer, A. T., Witt, A. A. \& Oh, D. The effect of mindfulnessbased therapy on anxiety and depression: a meta-analytic review. J. Consult Clin. Psychol. 78, 169-183 (2010).

31. Kuyken, W. et al. Efficacy of mindfulness-based cognitive therapy in prevention of depressive relapse: an individual patient data meta-analysis from randomized trials. JAMA Psychiatry 73, 565-574 (2016).

32. Goldberg, S. B. et al. Mindfulness-based cognitive therapy for the treatment of current depressive symptoms: a meta-analysis. Cogn. Behav. Ther. 48, 445-462 (2019).

33. Depression in adults: treatment and management: nice guideline. In development [GID-CGWAVE0725]: second consultation closed 12 June 2018. https://www.nice.org.uk/guidance/gid-cgwave0725/documents/htmlcontent-2 (National Institute for Health and Care Excellence, London, 2018).

34. Goyal, M. et al. Meditation programs for psychological stress and well-being: a systematic review and meta-analysis. JAMA Intern. Med. 174, 357-368 (2014).

35. Sevinc, G. et al. Strengthened hippocampal circuits underlie enhanced retrieval of extinguished fear memories following mindfulness training. Biol. Psychiatry 86, 693-702 (2019)

36. Goldin, P. R. et al. Group CBT versus MBSR for social anxiety disorder: a randomized controlled trial. J. Consult Clin. Psychol. 84, 427-437 (2016)

37. Khoury, B., Sharma, M., Rush, S. E. \& Fournier, C. Mindfulness-based stress reduction for healthy individuals: a meta-analysis. J. Psychosom. Res. 78 519-528 (2015).

38. Denkova, E., Zanesco, A. P., Rogers, S. L. \& Jha, A. P. Is resilience trainable? An initial study comparing mindfulness and relaxation training in firefighters. Psychiatry Res. 285, 112794 (2020).

39. Jha, A. P., Stanley, E. A., Kiyonaga, A., Wong, L. \& Gelfand, L. Examining the protective effects of mindfulness training on working memory capacity and affective experience. Emotion 10, 54-64 (2010).

40. Rooks, J. D., Morrison, A. B., Goolsarran, M., Rogers, S. L. \& Jha, A. P. "We Are Talking About Practice": the Influence of Mindfulness vs. Relaxation Training on Athletes' Attention and Well-Being over High-Demand Intervals. J. Cogn. Enhanc. 1, 141-153 (2017).

41. Dimidjian, S. et al. Considering meta-analysis, meaning, and metaphor: a systematic review and critical examination of "Third Wave" cognitive and behavioral therapies. Behav. Ther. 47, 886-905 (2016).

42. Dimidjian, S. \& Segal, Z. V. Prospects for a clinical science of mindfulness-based intervention. Am. Psychol. 70, 593-620 (2015).

43. Tang, Y.-Y., Hölzel, B. K. \& Posner, M. I. The neuroscience of mindfulness meditation. Nat. Rev. Neurosci. 16, 213-225 (2015).

44. Fox, K. C. R. et al. Functional neuroanatomy of meditation: a review and metaanalysis of 78 functional neuroimaging investigations. Neurosci. Biobehav. Rev. 65, 208-228 (2016).

45. Weiss, D. S. \& Marmar, C. R. Assessing Psychological Trauma And PTSD. 399-411 (Guilford Press, New York, 1997).

46. Spitzer, R. L., Kroenke, K., Williams, J. B. W. \& Löwe, B. A brief measure for assessing generalized anxiety disorder: the GAD-7. Arch. Intern. Med. 166 1092-1097 (2006).

47. Roberts, R. E. \& Vernon, S. W. The center for epidemiologic studies depression scale: its use in a community sample. Am. J. Psychiatry 140, 41-46 (1983).

48. Mingyuan, Z. \& Yanling, H. (ed). Handbook of Questionnaires for Psychiatry (Hunan Science and Technology Publisher, Changsha, 2015).

49. Lutz, A., Jha, A. P., Dunne, J. D. \& Saron, C. D. Investigating the phenomenological matrix of mindfulness-related practices from a neurocognitive perspective. Am. Psycho 70, 632-658 (2015).
50. Faul, F., Erdfelder, E., Buchner, A. \& Lang, A.-G. Statistical power analyses using G*Power 3.1: tests for correlation and regression analyses. Behav. Res. Methods 41, 1149-1160 (2009).

51. Busch, M. A., Maske, U. E., Ryl, L., Schlack, R. \& Hapke, U. Prävalenz von depressiver Symptomatik und diagnostizierter Depression bei Erwachsenen in Deutschland: Ergebnisse der Studie zur Gesundheit Erwachsener in Deutschland (DEGS1). Bundesgesundheitsblatt Gesundheitsforschung Gesundheitsschutz 56, 733-739 (2013).

52. Strine, T. W. et al. Depression and anxiety in the United States: findings from the 2006 Behavioral Risk Factor Surveillance. System. Psychiatr. Sen $\mathbf{5 9}$ 1383-1390 (2008).

53. Gotink, R. A., Meijboom, R., Vernooij, M. W., Smits, M. \& Hunink, M. G. M. 8-week Mindfulness Based Stress Reduction induces brain changes similar to traditional long-term meditation practice-A systematic review. Brain Cogn. 108, 32-41 (2016)

54. Williams, J. M. G. \& Kuyken, W. Mindfulness-based cognitive therapy: a promisingnew approach to preventing depressive relapse. Br. J. Psychiatry 200, 359-360 (2012).

55. Galante, J. et al. A mindfulness-based intervention to increase resilience tostress in university students (the Mindful Student Study): a pragmatic randomised controlled trial. Lancet Public Health 3, e72-e81 (2018).

56. Garland E. L., Hanley A. W., Baker A. K. \& Howard M. O. Biobehavioral Mechanisms of Mindfulness as a Treatment for Chronic Stress: An RDoC Perspective. Chronic Stress (Thousand Oaks) 1 (2017).

57. Berenbaum, H. A taxonomy of emotional disturbances. Clin. Psychol. 10, 206-226 (2003)

58. Mennin, D. \& Farach, F. Emotion and evolving treatments for adult psychopathology. Clin. Psychol. Sci. Pr. 14, 329-352 (2007).

59. Gross, J. J. \& Muñoz, R. F. Emotion regulation and mental health. Clin. Psychol. Sci. Pr. 2, 151-164 (1995).

60. Garland, E. L., Farb, N. A., Goldin, P. \& Fredrickson, B. L. Mindfulness broadens awareness and builds eudaimonic meaning: a process model of mindful positive emotion regulation. Psychol. Inq. 26, 293-314 (2015).

61. Farb, N. A. S., Anderson, A. K. \& Segal, Z. V. The mindful brain and emotion regulation in mood disorders. Can. J. Psychiatry 57, 70-77 (2012).

62. Goldin, P. R. \& Gross, J. J. Effects of mindfulness-based stress reduction (MBSR) on emotion regulation in social anxiety disorder. Emotion 10, 83-91 (2010).

63. Guendelman, S., Medeiros, S. \& Rampes, H. Mindfulness and emotion regulation: insights from neurobiological, psychological, and clinical studies. Front. Psychol. 8, 220 (2017).

64. Chiesa, A., Serretti, A. \& Jakobsen, J. C. Mindfulness: top-down or bottom-up emotion regulation strategy? Clin. Psychol. Rev. 33, 82-96 (2013).

65. van der Velden, A. M. et al. A systematic review of mechanisms of change in mindfulness-based cognitive therapy in the treatment of recurrent major depressive disorder. Clin. Psychol. Rev. 37, 26-39 (2015).

66. van Vugt, M. K. Hitchcock, P., Shahar, B. \& Britton, W. The effects of mindfulness-based cognitive therapy on affective memory recall dynamics in depression: a mechanistic model of rumination. Front. Hum. Neurosci. 6, 257 (2012).

67. Watkins, E. R. Constructive and unconstructive repetitive thought. Psychol. Bull. 134, 163-206 (2008).

68. Smallwood, J. \& Schooler, J. W. The science of mind wandering: empirically navigating the stream of consciousness. Annu. Rev. Psychol. 66, 487-518 (2015).

69. Kral, T. R. A. et al. Impact of short- and long-term mindfulness meditation training on amygdala reactivity to emotional stimuli. Neuroimage 181, 301-313 (2018).

70. Parsons, C. E., Crane, C., Parsons, L. J., Fjorback, L. O. \& Kuyken, W. Home practice in Mindfulness-Based Cognitive Therapy and Mindfulness-Based Stress Reduction: a systematic review and meta-analysis of participants' mindfulness practice and its association with outcomes. Behav. Res. Ther. 95 29-41 (2017).

71. Spijkerman, M. P. J., Pots, W. T. M. \& Bohlmeijer, E. T. Effectiveness of online mindfulness-based interventions in improving mental health: a review and meta-analysis of randomised controlled trials. Clin. Psychol. Rev. 45, 102-114 (2016).

72. Crane, R. S. \& Kuyken, W. The implementation of mindfulness-based cognitive therapy: learning from the UK health service experience. Mindfulness 4, 246-254 (2013). 